\title{
Video Article \\ Laser Capture Microdissection of Enriched Populations of Neurons or Single Neurons for Gene Expression Analysis After Traumatic Brain Injury
}

\author{
Deborah R. Boone ${ }^{1}$, Stacy L. Sell ${ }^{1}$, Helen Lee Hellmich ${ }^{1}$ \\ ${ }^{1}$ Department of Anesthesiology, University of Texas Medical Branch \\ Correspondence to: Helen Lee Hellmich at hhellmic@utmb.edu \\ URL: https://www.jove.com/video/50308 \\ DOI: doi: $10.3791 / 50308$
}

Keywords: Neuroscience, Issue 74, Neurobiology, Medicine, Biomedical Engineering, Anatomy, Physiology, Cellular Biology, Molecular Biology, Genetics, Surgery, Anesthesiology, Micromanipulation, Microdissection, Laser Capture Microdissection, LCM, Investigative Techniques, traumatic brain injury, TBI, hippocampus, Fluoro-Jade, gene expression analysis, gene expression, neurons, animal model

Date Published: 4/10/2013

Citation: Boone, D.R., Sell, S.L., Hellmich, H.L. Laser Capture Microdissection of Enriched Populations of Neurons or Single Neurons for Gene Expression Analysis After Traumatic Brain Injury. J. Vis. Exp. (74), e50308, doi:10.3791/50308 (2013).

\section{Abstract}

Long-term cognitive disability after TBI is associated with injury-induced neurodegeneration in the hippocampus-a region in the medial temporal lobe that is critical for learning, memory and executive function. ${ }^{1,2}$ Hence our studies focus on gene expression analysis of specific neuronal populations in distinct subregions of the hippocampus. The technique of laser capture microdissection (LCM), introduced in 1996 by Emmert-Buck, et al., ${ }^{3}$ has allowed for significant advances in gene expression analysis of single cells and enriched populations of cells from heterogeneous tissues such as the mammalian brain that contains thousands of functional cell types. ${ }^{4}$ We use LCM and a well established rat model of traumatic brain injury (TBI) to investigate the molecular mechanisms that underlie the pathogenesis of TBI. Following fluidpercussion TBI, brains are removed at pre-determined times post-injury, immediately frozen on dry ice, and prepared for sectioning in a cryostat. The rat brains can be embedded in OCT and sectioned immediately, or stored several months at $-80^{\circ} \mathrm{C}$ before sectioning for laser capture microdissection. Additionally, we use LCM to study the effects of TBI on circadian rhythms. For this, we capture neurons from the suprachiasmatic nuclei that contain the master clock of the mammalian brain. Here, we demonstrate the use of LCM to obtain single identified neurons (injured and degenerating, Fluoro-Jade-positive, or uninjured, Fluoro-Jade-negative) and enriched populations of hippocampal neurons for subsequent gene expression analysis by real time PCR and/or whole-genome microarrays. These LCM-enabled studies have revealed that the selective vulnerability of anatomically distinct regions of the rat hippocampus are reflected in the different gene expression profiles of different populations of neurons obtained by LCM from these distinct regions. The results from our single-cell studies, where we compare the transcriptional profiles of dying and adjacent surviving hippocampal neurons, suggest the existence of a cell survival rheostat that regulates cell death and survival after TBI.

\section{Video Link}

The video component of this article can be found at https://www.jove.com/video/50308/

\section{Introduction}

Gene expression analysis of heterogeneous tissues has always been problematic; this is particularly true in the mammalian brain, which has approximately 5,000 different cell types. ${ }^{4}$ Prior to the development of the laser capture microdissection (LCM) technique, genomic studies of the effects of TBI in vivo were based on analysis of gene expression in a mixed population of brain cells comprised, not only of different neuronal cell types, but also of supporting glial and immunomodulatory cells. The resulting complex gene expression profiles obtained from these heterogeneous tissues, and the often conflicting patterns of injury-induced cellular signals, may be one explanation for the failure in human clinical trials of therapeutic strategies proven effective in pre-clinical studies of TBI. ${ }^{5}$

To obtain a clear understanding of injury-induced gene expression in vulnerable populations of neurons from the rat hippocampus, we adopted the technique of LCM, first reported by Emmert-Buck et al. ${ }^{3}$ Subsequently, we modified and optimized this microdissection technique for efficient capture of enriched populations of neurons and single neurons for mRNA profiling using quantitative real time PCR and microarray analysis. To maintain the integrity of mRNA in frozen sections of brain tissue for genomic analysis, we modified existing protocols for fixing, staining and rapid capture of neurons from frozen rat brain sections. For identification and isolation of injured and dying hippocampal neurons, we also optimized the Fluoro-Jade staining technique for LCM. Fluoro-Jade does not distinguish between apoptotic and necrotic cell death. Thus, all types of degenerating neurons can be detected by this stain. 6,7

Here, we describe the protocol used in our laboratory to obtain pools of single dying or surviving neurons as well as swaths of enriched populations of distinct neuronal cell types (i.e. CA1-CA3 neurons for gene expression analysis after TBI). The procedure for fluid percussion brain injury performed in our laboratory is described in detail in Shimamura et al., ${ }^{8}$ and is very similar to the lateral fluid-percussion injury protocol for mice published in JoVE by Alder et al. ${ }^{9}$ Since the LCM technique has been shown to have minimal or no effect on the integrity of DNA, RNA and protein in tissues, this is an excellent tool for molecular and protein analysis of defined cell types. 


\section{Surgical Procedures and Fluid Percussion TBI}

1. All animal experiments are first approved by the Institutional Animal Care and Use Committee of the University of Texas Medical Branch, Galveston, Texas and the National Institutes of Health Guide for the Care and Use of Laboratory Animals (8th Edition, National Research Council).

2. Rats (adult male Sprague-Dawley rats, $400-500 \mathrm{~g}$ obtained from vendor Charles Rivers, Portland, Maine) are housed two per cage and provided food and water ad libitum in a vivarium with these constant conditions: light cycle $(600 \mathrm{hr}$ to $1,800 \mathrm{hr})$, temperature $\left(21^{\circ} \mathrm{C}\right.$ to $\left.23{ }^{\circ} \mathrm{C}\right)$, and humidity $(40 \%$ to $50 \%)$ one week prior to use.

3. Anesthetize rats with $4 \%$ isoflurane, intubate, and mechanically ventilate (NEMI Scientific; New England Medical Instruments, Medway, MA) the rats with $1.5-2.0 \%$ isoflurane in oxygen: air (70:30) and prepare them for parasagittal fluid-percussion injury as previously described. ${ }^{8,10}$

4. Sacrifice rats at the appropriate time point after injury depending on the experimental design. Quickly remove brains, freeze immediately on dry ice, and store at $-80^{\circ} \mathrm{C}$ in a $50 \mathrm{ml}$ tube or proceed immediately to embed in OCT for frozen sectioning.

\section{Sectioning and Staining of Rat Brain}

1. Brain tissue that is not used immediately can be kept at $-80^{\circ} \mathrm{C}$ for up to one month if kept at a constant temperature. Brains that are frozen at $-80^{\circ} \mathrm{C}$, then thawed to $-20^{\circ} \mathrm{C}$ for sectioning, and then re-frozen do not yield quality RNA after the second thawing. Once the brain is thawed, mounted in OCT and sectioned, slides should be stained within $24 \mathrm{hr}$ and used for LCM within 30 min to one hour of staining. This will ensure good quality RNA. Following LCM, captured cells on LCM caps can be stored in lysis buffer at $-80^{\circ} \mathrm{C}$ for up to one week, but RNA should be isolated in a timely manner to ensure the highest quality.

2. Prior to sectioning the brain, wipe the cryostat with RNase-Zap and clean the brushes with ETOH (replace the disposable blade between each brain).

3. Retrieve the brain in the $50 \mathrm{ml}$ tube from the $-80^{\circ} \mathrm{C}$ freezer; place it into the cryostat at a temperature of $-22{ }^{\circ} \mathrm{C}$ and thaw in tube for approximately $10 \mathrm{~min}$. Remove the brain from the tube and place onto the stage on gauze, ventral side up.

4. Using a razor blade, slice the brain to remove the posterior portion of the brain just rostral to the cerebellum and the anterior portion at the optic chiasm. Fill a cryomold with OCT mounting medium (Tissue Tek), and place the brain into the mold with the anterior side down. Allow the brain tissue to freeze in the mounting medium until it turns white (approximately $10 \mathrm{~min}$ ).

5. Freeze the specimen disc (Tissue Tek) onto the brain with OCT. Remove the brain from the mold. Insert the brain into the specimen head and tighten the screws.

6. Insert a disposable, low profile blade (Fisher Scientific) into the knife holder and tighten the lever down.

7. Begin slicing the brain at $20 \mu \mathrm{m}$ to remove the outer layer of OCT. Once the hippocampal region is reached, set the micron dial to 10 . Collect coronal serial sections by placing a glass slide or plus-glass slide onto the tissue section (Fisher Scientific). Slides are preserved at $-20{ }^{\circ} \mathrm{C}$ in an RNase-free staining rack until sectioning is complete.

8. To remove all RNases from glassware where tissue sections are processed, wipe down all staining containers and graduated cylinders with Eliminase (Fisher Scientific) and rinse in Milli Q water. Prepare all solutions with RNase-free water and filter the cresyl violet (Sigma-Aldrich) and Fluoro-Jade (Histo-chem) stain with a $0.2 \mu \mathrm{m}$ filter prior to use.

9. Thaw brain sections at RT for $30 \mathrm{sec}$ and fix in $75 \% \mathrm{ETOH}(1 \mathrm{~min})$.

10. For LCM of single injured neurons after fixation, rinse slides in RNase-free water (1 min), counterstain with $1 \%$ cresyl violet (15-20 sec), rinse in RNase-free water $(2 \times 30 \mathrm{sec})$, stain with Fluoro-Jade $(4 \mathrm{~min})$, rinse in RNase-free water $(3 \times 1 \mathrm{~min})$, dehydrate with $95 \%$ ETOH made from RNase-free water (30 sec), 100\% ETOH (30 sec), and xylene ( $2 \times 3 \mathrm{~min})$.

11. For LCM of swaths of neurons after fixation, rinse slides in RNase-free water (1 min), stain with $1 \%$ cresyl violet ( 1 min), rinse in RNase-free water for $(3 \times 1 \mathrm{~min})$, dehydrate with $95 \% \mathrm{ETOH}(2 \times 30 \mathrm{sec}), 100 \% \mathrm{ETOH}(2 \times 30 \mathrm{sec})$, and xylene $(2 \times 3 \mathrm{~min})$.

12. Air dry all sections in a fume hood for $15 \mathrm{~min}$ prior to LCM. Slides can be stored, section sides up, in a slide box lined with dessicant, if they need to be transferred from room to room. However, optimal results are obtained if LCM is performed immediately after sections are dry. LCM should be limited from $30 \mathrm{~min}$ to $1 \mathrm{hr}$. In the next section, we describe first how to capture continuous swaths of cells, which is easiest to master for those who are learning this technique. Then we describe how to precisely capture single neurons. In our procedure, we identify dying neurons by their affinity for Fluoro-Jade-a marker of degenerating neurons. Fluoro-Jade-negative cells are presumed to be surviving neurons.

\section{Laser Capture Microdissection (LCM)}

\section{LCM of swaths of enriched populations of hippocampal neurons}

1. LCM is performed using a PixCell lle microscope with an infrared diode laser (Life Technologies).

2. Adjust the center joystick to the vertical position. Rotate and lock the $4 \mathrm{X}$ objective into position.

3. Place a slide at the center of the microscope stage and manually adjust until the region of the hippocampus is in view. Use coarse and fine focus adjustments to bring the image into focus.

4. Activate the vacuum chuck by pressing the vacuum button on the controller. Rotate and lock the 10X objective into place. Focus again with coarse and fine adjustments.

5. Load the CapSure Macro LCM caps (Life Technologies) into the CapSure cassette module and position a cap at the load line position. Rotate the Cap Placement arm and position around the cap. Raise the Cap Placement arm to remove the CapSure cap from the cassette module.

6. Rotate the Cap Placement arm over the slide and lower the arm down over the slide; this will place the cap onto the slide. Adjust the fine focus and move the joystick to check if cells are inside the black circle on the cap. All cell captures should be inside this black circle. 
7. Set the laser parameters. First, press the laser-enable button on the controller. The laser target spot will be visible as a pink dot in the field of view on the computer monitor.

8. Set the laser spot size to small $(7.5 \mu \mathrm{m})$ to focus the laser and adjust the power and duration to $65-75 \mathrm{~mW}$, for $1.0-2.0 \mathrm{msec}$ as needed for optimal cell capture.

9. To test the laser, use the joystick to move the laser spot to an area where there are no cells to pick up. Fire the laser using the thumb switch. Use the joystick to move the laser spot away from the melted polymer spot and check to see if a visible dark ring surrounds a clear area where the laser was fired.

10. To capture cells, use the joystick to move the laser spot over the area of cells to capture. Fire the laser using the thumb switch. Move the joystick while firing the laser to capture a large area of cells. When the laser is fired it melts the thermoplastic polymer film on the cap to the surface of the target cells. The polymer absorbs the laser radiation, thus not altering the RNA, DNA or protein ensuring the integrity of the sample for future molecular applications.

11. After firing all cells of interest, lift the Cap Placement arm. The captured cells will separate from the tissue section and adhere to the CapSure cap. The remaining tissue and missing cells will be visible in the field of view. The cells on the cap can also be viewed by placing the cap on an empty portion of the slide.

12. Lift the Cap Placement arm and rotate it to the Cap Unload Station. Lower the cap into the station and rotate the Cap Placement arm back into its previous position.

13. Slide the CapSure insertion tool along the platform and onto the cap. Lift the tool from the platform. The cap will remain attached. Lightly touch the cap to the CapSure clean pad to clean away unwanted tissue.

14. Place the cap into a $0.5 \mathrm{ml}$ RNase-free tube filled with $100 \mu \mathrm{l}$ of lysis buffer obtained from the RNAqueous Micro-Isolation Kit. Immediately vortex the cap for $30 \mathrm{sec}$ to lyse the cells. Incubate the samples at $42{ }^{\circ} \mathrm{C}$ for $30 \mathrm{~min}$ and freeze at $-80^{\circ} \mathrm{C}$ until RNA isolation.

\section{LCM of single FJ+ neurons in the hippocampus}

15. To capture single cells, load CapSure HS LCM caps into the CapSure cassette module and position a cap at the load line position. Rotate the Cap Placement arm and position it around the cap. Raise the Cap Placement arm to remove the CapSure cap from the cassette module.

16. Set the laser spot size to small $(7.5 \mu \mathrm{m})$ to focus the laser and adjust the laser power and duration to $65-75 \mathrm{~mW}$ for 0.45 to $0.50 \mathrm{msec}$ for optimal cell capture of single cells.

17. Rotate the Cap Placement arm over the slide and lower the arm down toward the slide. This will place the cap onto the slide. The Capsure HS cap surface sits elevated from the sample during LCM.

18. Use the joystick to move the laser over single FJ+ cells. All cells being captured must be inside the small black ring on the cap. Fire the laser using the thumb switch.

19. When all cells within the black circle area have been fired with the laser, lift the Cap Placement arm. The captured cells will separate from the tissue section and adhere to the CapSure HS cap.

20. Place another slide on the microscope stage and collect FJ+ cells until the HS cap is full. Place the cap into a $0.5 \mathrm{ml}$ RNase-free tube filled with $40-50 \mu \mathrm{l}$ of lysis buffer. Immediately vortex the cap for $30 \mathrm{sec}$ to lyse the cells. Incubate the samples at $42{ }^{\circ} \mathrm{C}$ for 30 min and freeze at -80 ${ }^{\circ} \mathrm{C}$ until RNA isolation.

\section{Total RNA Isolation From LCM Samples (This section will just be described in the video presentation)}

1. Total RNA from cells is isolated using the RNAqueous-Micro Kit (Ambion) following the manufacturer's protocols. All reagents and wash solutions are provided in this kit. Pre-wet the micro filter cartridge assembly by adding $30 \mu$ l of lysis solution buffer to the filter. After 5 min, centrifuge the filter for $30 \mathrm{sec}$ at $10,000 \times \mathrm{g}$.

2. Add $3 \mu$ l of LCM additive to the sample lysate, pipette to mix, and centrifuge.

3. Add $52 \mu \mathrm{l}$ of $100 \% \mathrm{ETOH}$ (1/2 volume) to the sample lysate; pipette to mix, and transfer the lysate to the filter column.

4. Centrifuge the filter column at $10,000 \times \mathrm{g}$ for $1 \mathrm{~min}$ to bind the RNA to the column. If there are multiple caps for one sample, spin each lysate through the same column separately.

5. Complete the RNA isolation procedure as described in the RNAqueous Micro kit.

6. Perform DNase treatment and DNase inactivation of LCM RNA samples as described in the kit, transfer the RNA to a RNase-free tube and store at $-80^{\circ} \mathrm{C}$.

\section{RNA Quantification and Downstream Applications}

Assessment of RNA quality and quantification is performed with an Agilent Bioanalyzer using reagents from the Pico Kit (Agilent Technologies, Santa Clara, CA) following the manufacturer's protocol.

1. Total RNA is analyzed on an Agilent Bioanalyzer 2100 (Agilent Technologies). The software evaluates the concentration and the integrity of the RNA sample by assigning an RNA Integrity Number (RIN) to each sample ranging from 1 to 10. 1-5 indicates degraded RNA and 7-10 indicates good quality RNA.

2. Non-degraded intact RNA will be used for Real-Time PCR; individual Taqman assays or RT2 profiler arrays using Sybr-green chemistry. The RNA can also be used for mRNA microarray analysis and microRNA microarray analysis.

\section{Representative Results}

In our first LCM study, we were able to show that functionally distinct subregions (CA1 and CA3) of the rat hippocampus have gene expression profiles that reflect their vulnerability to TBI. ${ }^{8}$ Figure 1A-1C shows laser capture of hippocampal pyramidal neurons (CA1-CA3), dentate gyrus neurons and SCN neurons before, and after LCM. Clean captures of pyramidal, granule or SCN neurons, respectively, are shown on the macro 
caps. Figure 2A-2B illustrates dying, Fluoro-Jade positive pyramidal neurons and surviving, Fluoro-Jade negative pyramidal neurons before and after LCM. The clean capture of the dying or surviving neurons is shown by viewing the captured cells on the LCM caps.

After LCM, the total RNA can be used for a diverse array of molecular studies including quantitative real-time PCR analysis of gene expression using TaqMan or SYBR green chemistries (Figure 3A), small focused PCR arrays with SYBR green probes (Figure 3B) or whole genome microarray studies (Figure $3 \mathrm{C}$ ).
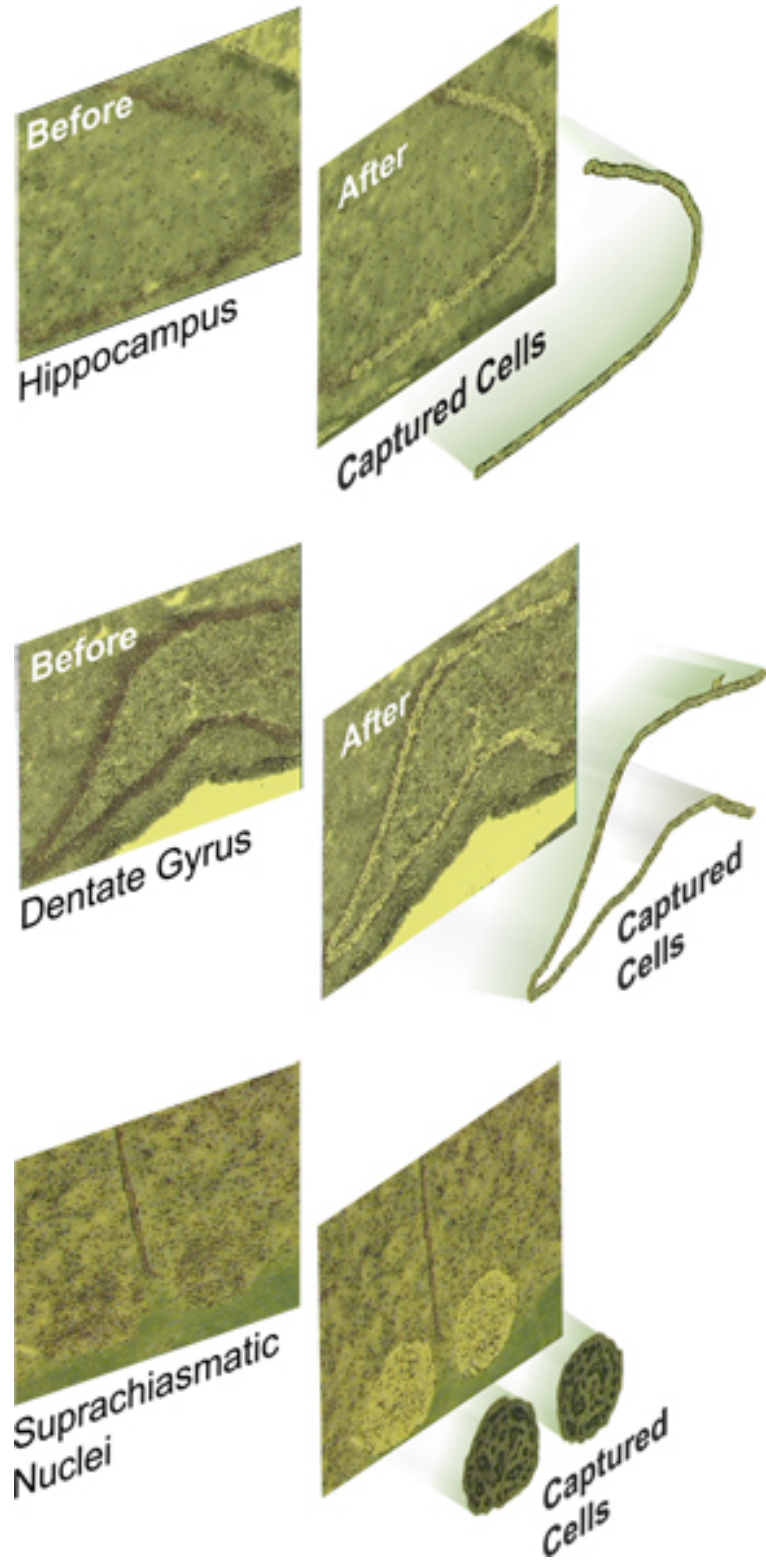

Figure 1. Laser capture microdissection of swaths of defined cell populations from the rat brain. Frozen $10 \mu \mathrm{m}$ coronal sections of the rat brain are fixed in ethanol, stained with a nissl stain (cresyl violet) and prepared for LCM. Shown are pictures of the rat hippocampus and suprachiasmatic nucleus before and after LCM and the captured cells as visualized on the macro caps. A. Pyramidal neurons from the CA1-CA3 subfields of the rat hippocampus. B. Granule cells in the hippocampal dentate gyrus. C. Bilateral suprachiasmatic nuclei located on either side of the third ventricle and located above the optic chiasma. Click here to view larger figure. 

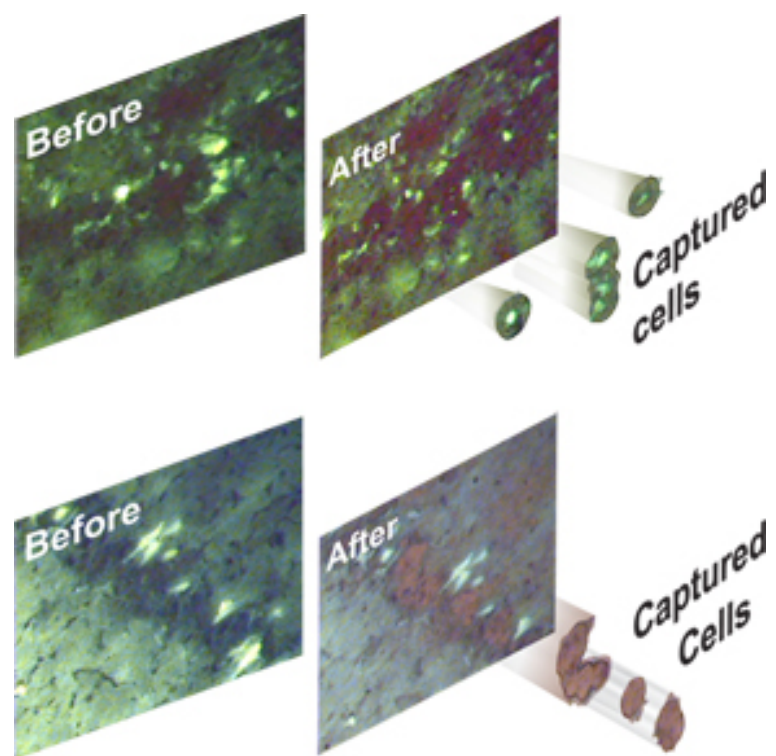

Figure 2. Laser capture microdissection of single neurons from rat hippocampus. Shown are A. Degenerating, Fluoro-Jade-positive (stained) hippocampal CA3 neurons and B. Surviving, Fluoro-Jade-negative (unstained) CA3 neurons before and after LCM. Captured cells are visualized. Click here to view larger figure.
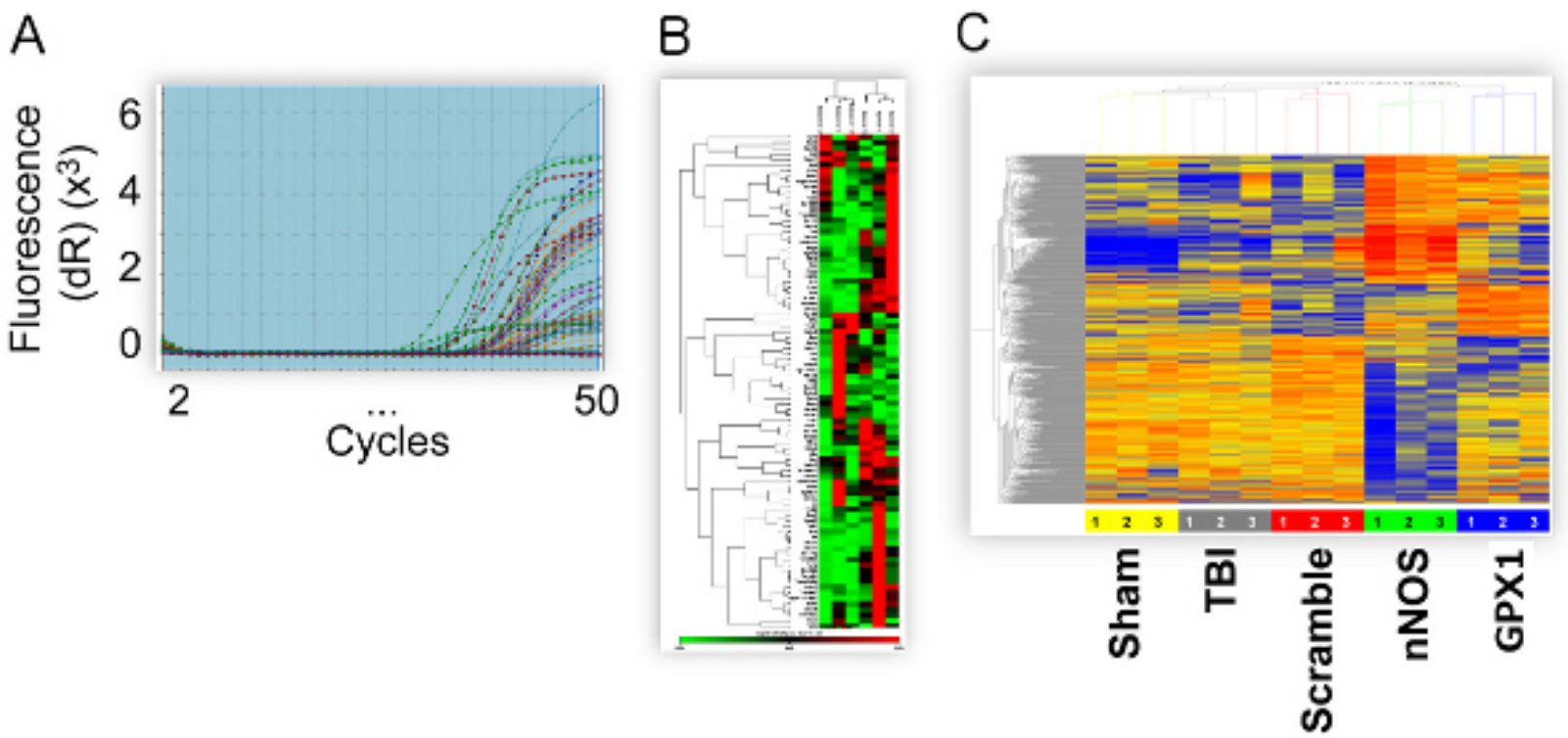

Figure 3. Gene expression analysis of total RNA isolated from laser captured neurons. A. Quantitative real time PCR data of circadian clock gene expression in SCN. B. Heatmap of gene expression in dying and surviving hippocampal neurons showing expression of apoptosisrelated genes using focused PCR arrays. C. Agilent whole-genome microarray analysis of gene expression in hippocampal CA1-CA3 neurons from rats that received sham injury, TBI or TBI plus a recombinant adeno-associated siRNA virus designed to knockdown expression of genes induced by brain injury (neuronal nitric oxide synthase and glutathione peroxidase-1). Click here to view larger figure.

\section{Discussion}

This LCM technique has enabled us to make significant advances in understanding the molecular mechanisms of TBI..$^{8,10,11,12,13}$ We were the first investigators to utilize this technique to demonstrate that distinct subregions of the rat hippocampus have gene expression profiles that correlate with their selective vulnerability to injury. Our studies showed that we could quantify gene expression, by qPCR, from as few as 10 laser captured cells and that we could perform genome-wide microarray analysis from as few as 600 captured cells. LCM would be a valuable tool in similar studies of other brain regions that are known to be implicated in diverse neurological and neuropsychiatric disorders. For example, studies using LCM have shed light into the pathogenesis of Parkinson's disease in dopamine neurons of the substantia nigra, ${ }^{14}$ and aided genome-wide profiling studies of the nucleus accumbens, which is involved in reward circuits implicated in substance abuse disorders. ${ }^{15}$

Neuronal heterogeneity is reflected at the genome level ${ }^{16}$ and may contribute to the lack of success of experimental treatments for TBI in clinical trials. Thus, our goal is to utilize this technique to investigate the critical elements influencing neuronal survival after TBI. Our recent genomewide profiling study of dying and adjacent surviving hippocampal neurons after TBI suggested that a cellular rheostat that reflects the ratio of the expression levels of cell survival to cell death genes regulates cell fate after TBI. These ongoing studies will contribute to the design and 
development of pharmacotherapeutic strategies that can positively influence the cell survival rheostat. Furthermore, we currently use LCM to track and monitor the effects of potential therapeutic drug treatments in hippocampal neurons after TBI. Thus, our studies demonstrate that given careful RNase-free handling techniques and some modifications of existing protocols, it is possible to obtain high quality RNA samples from LCM for accurate quantitative gene expression analysis.

However there are a few pitfalls associated with LCM techniques. For example, collecting only neuronal cells without any microglia contamination can be almost impossible. In the pyramidal cell layer of the hippocampus it has been estimated that $95 \%$ of the cells are neurons with $90 \%$ of this population to be pyramidal cells and $10 \%$ interneurons, leaving a small percentage of glial and other cell types. ${ }^{8,17,18}$ In our studies we use Fluoro-jade a fluorescent dye that specifically labels only injured neurons in brain tissue. A different stain that is a marker for GFAP would be necessary to stain microglia. ${ }^{19}$ Collecting only the Fluoro-jade stained single neuronal bodies ensures a more homogenous population of neurons. Another common problem that may require troubleshooting is that a circle is not defined when the laser is fired. If this occurs, it is necessary to check the laser settings and adjust the power and duration as necessary. Also it is important to ensure that the cap is placed flat on the tissue and seated correctly in the arm. Referring to the LCM technical manual or calling technical support will sometimes be necessary. Following LCM and RNA isolation, the quality of the RNA should always be assessed using a bioanalyzer prior to any gene expression analysis.

There are several types of laser capture instruments currently on the market including laser cutting (Life Technologies, Leica Microsystems) and laser catapulting (Zeiss) instruments. Our LCM system works well for capturing small numbers of cells. Other high-throughput and more automated systems may be more suitable for obtaining larger numbers of cells for genomic and particularly proteomic analysis. Indeed, LCM using these automated systems has great potential for analysis of protein expression in identified cells or enriched populations of cells. ${ }^{20}$ Furthermore, LCM can facilitate gene expression analysis of immunolabeled cells, ${ }^{21}$ allowing us to investigate gene expression in defined cell types regardless of the complexity in the most heterogeneous tissues. Thus, LCM is an excellent tool for cutting-edge molecular studies of single or enriched populations of cells.

\section{Disclosures}

The authors declare that they have no conflicts of interest.

\section{Acknowledgements}

This work is funded by R01 NS052532 (to HLH), the Moody Foundation, and the Department of Anesthesiology. We thank Laurie Bolding, Christine Courteau Butler and Christy Perry for their editorial assistance, and Christy Perry for layout and excellent production of all figures, tables and artwork.

\section{References}

1. Squire, L.R., Stark, C.E., \& Clark, R.E. The medial temporal lobe. Annual Review of Neuroscience. 27, 279-306 (2004).

2. Bast, T. Toward an integrative perspective on hippocampal function: from the rapid encoding of experience to adaptive behavior. Rev. Neurosci. 18 (3-4), 253-281 (2007).

3. Emmert-Buck, M.R., Bonner, R.F., et al. Laser capture microdissection. Science. 274 (5289), 998-1001 (1996).

4. Bota, M., Dong, H.W., \& Swanson, L.W. From gene networks to brain networks. Nat. Neurosci. 6 (8), 795-799 (2003).

5. Schouten, J.W. Neuroprotection in traumatic brain injury: a complex struggle against the biology of nature. Curr. Opin. Crit. Care. 13 (2), 134-142 (2007).

6. Schmued, L.C., Albertson, C., \& Slikker, W., Jr. Fluoro-Jade: a novel fluorochrome for the sensitive and reliable histochemical localization of neuronal degeneration. Brain Research. 751 (1), 37-46 (1997).

7. Ye, X., Carp, R.I., Schmued, L.C., \& Scallet, A.C. Fluoro-Jade and silver methods: application to the neuropathology of scrapie, a transmissible spongiform encephalopathy. Brain Res. Brain Res. Protoc. 8 (2), 104-112 (2001).

8. Shimamura, M., Garcia, J.M., Prough, D.S., \& Hellmich, H.L. Laser capture microdissection and analysis of amplified antisense RNA from distinct cell populations of the young and aged rat brain: effect of traumatic brain injury on hippocampal gene expression. Mol. Brain Res. 17 (1), 47-61 (2004).

9. Alder, J., Fujioka, W., Lifshitz, J., Crockett, D.P., \& Thakker-Varia, S. Lateral Fluid Percussion: Model of Traumatic Brain Injury in Mice. J. Vis. Exp. (54), e3063, doi:10.3791/3063 (2011).

10. Shimamura, M., Garcia, J.M., et al. Analysis of long-term gene expression in neurons of the hippocampal subfields following traumatic brain injury in rats. Neuroscience. 131 (1), 87-97 (2005).

11. Shah, S.A., Prough, D.S., Garcia, J.M., DeWitt, D.S., \& Hellmich, H.L. Molecular correlates of age-specific responses to traumatic brain injury in mice. Exp. Gerontol. 41 (11), 1201-5 (2006).

12. Hellmich, H.L., Garcia, J.M., et al. Traumatic brain injury and hemorrhagic hypotension suppress neuroprotective gene expression in injured hippocampal neurons. Anesthesiology. 102 (4), 806-814 (2005).

13. Rojo, D.R., Prough, D.S., et al. Influence of stochastic gene expression on the cell survival rheostat after traumatic brain injury. PLoS One. 6 (8), e2311 (2011).

14. Elstner, M., Morris, C.M., et al. Expression analysis of dopaminergic neurons in Parkinson's disease and aging links transcriptional dysregulation of energy metabolism to cell death. Acta Neuropathol. 122 (1), 75-86 (2011).

15. Chen, H., Liu, Z., et al. Genome-Wide Gene Expression Profiling of Nucleus Accumbens Neurons Projecting to Ventral Pallidum Using both Microarray and Transcriptome Sequencing. Front. Neurosci. 5, 98 (2011).

16. Lein, E.S., Hawrylycz, M.J., et al. Genome-wide atlas of gene expression in the adult mouse brain. Nature. 445 (7124), 168-176 (2007).

17. Amaral, D.G., Ishizuka, N., \& Claiborne, B. Neurons, numbers and the hippocampal network. Prog. Brain Res. 83, 1-11 (1990). 
18. Zeng, Y.C., Bongrani, S., et al. Effect of long-term treatment with L-deprenyl on the age-dependent microanatomical changes in the rat hippocampus. Mech. Ageing Dev. 79 (2-3), 169-185 (1995).

19. Schmued, L.C. \& Hopkins, K.J. Fluoro-Jade: novel fluorochromes for detecting toxicant-induced neuronal degeneration. Toxicol. Pathol. 28 (1), 91-99 (2000).

20. Banks, R.E., Dunn, M.J., et al. The potential use of laser capture microdissection to selectively obtain distinct populations of cells for proteomic analysis-- preliminary findings. Electrophoresis. 20 (4-5), 689-700 (1999).

21. Fend, F., Emmert-Buck, M.R., et al. Immuno-LCM: laser capture microdissection of immunostained frozen sections for mRNA analysis. Am. J. Pathol. 154 (1), 61-66 (1999). 